\title{
Vibrational Relaxation Dynamics of the Core and Outer Part of Proton-Hydration Clusters
}

\author{
Oleksandr O. Sofronov**i] and Huib J. Bakker ${ }^{\circledR}$ \\ AMOLF, Science Park 104, 1098 XG Amsterdam, The Netherlands \\ Supporting Information
}

ABSTRACT: We study the ultrafast relaxation dynamics of hydrated proton clusters in acetonitrile using femtosecond mid-infrared pump-probe spectroscopy. We observe a strong dependence of transient absorption dynamics on the frequency of excitation. When we excite the $\mathrm{OH}$ vibrations with frequencies $\leq 3100 \mathrm{~cm}^{-1}$, we observe an ultrafast energy relaxation that leads to the heating of the local environment of the proton. This response is assigned to the $\mathrm{OH}$ vibrations of the water molecules in the core of the hydrated proton cluster. When we excite with frequencies $\geq 3200 \mathrm{~cm}^{-1}$, we observe a relatively slow vibrational relaxation with a $T_{1}$ time constant ranging from $0.22 \pm 0.04$ ps at $\nu_{\text {ex }}=3200 \mathrm{~cm}^{-1}$ to $0.37 \pm 0.02$ ps at $\nu_{\text {ex }}=3520 \mathrm{~cm}^{-1}$.

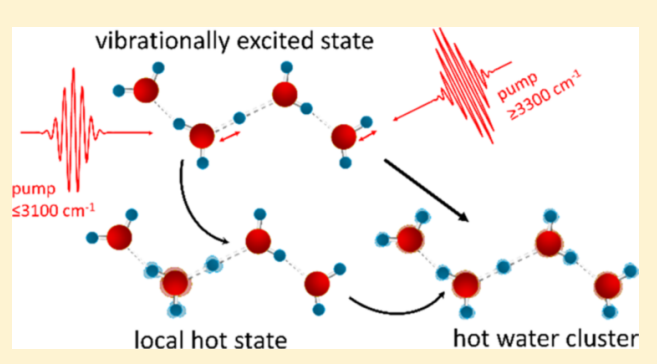

We assign this response to water molecules in the outer part of the hydrated proton cluster.

\section{INTRODUCTION}

The proton $\left(\mathrm{H}^{+}\right)$plays a key role in various chemical processes in aqueous media. ${ }^{1-4}$ For the protons in liquid water different solvation structures have been proposed, including the $\mathrm{H}_{3} \mathrm{O}^{+}\left(\mathrm{H}_{2} \mathrm{O}\right)_{3}$ Eigen cation, the $\mathrm{H}_{5} \mathrm{O}_{2}^{+}$Zundel cation, and the so-called asymmetric Zundel as an intermediate geometry of the first two. These structures are based on cryogenic photodissociation vibrational spectroscopy experiments of small protonated water clusters, ${ }^{5,6}$ and molecular dynamics simulations. $^{7-9}$ In the cluster experiments, Eigen and Zundel cation structures have been identified from the central frequencies of the $\mathrm{OH}$-stretch vibrations. However, quantitative infrared spectroscopy ${ }^{10,11}$ and photodissociation spectroscopy of large protonated water clusters ${ }^{5,12,13}$ as well as molecular dynamics simulations ${ }^{9,14-16}$ show that the water molecules in the second solvation shell of the proton also possess different properties from the molecules in bulk water, which implies that the Eigen and Zundel cations only represent the central cores of the proton-hydration structures in liquid water.

Infrared spectroscopy is a powerful technique for studying strongly hydrogen-bonded systems like the hydrated proton because the vibrational frequency of the stretch vibration of the hydrogen-bond donating group strongly depends on the strength of the donated hydrogen bond. ${ }^{4,17} \mathrm{~A}$ complication is that strong hydrogen bonding leads to extremely broad absorption bands for the $\mathrm{OH}$-stretch vibration. As a result, the hydrated proton in liquid water shows nearly continuous absorption spanning from 1000 to $3600 \mathrm{~cm}^{-1} \cdot{ }^{18-20}$ Recent ab initio molecular dynamics simulations show that the large width of the absorption band largely results from the heterogeneity of the hydrogen bonds, in particular the difference in hydrogen-bond strength between the water molecules in the first and the second hydration shells. ${ }^{21}$
Femtosecond infrared (fs-IR) spectroscopy provides information on the relaxation dynamics and can thereby help in the assignment of the different regions of the absorption spectrum of the $\mathrm{OH}$-stretch vibrations of the hydrated proton. fs-IR spectroscopy has recently been used to study the properties of hydrated proton clusters in acetonitrile. ${ }^{22-24}$ Acetonitrile as a weak polar solvent is a very suitable matrix for hydrated proton clusters.

In ref 23 it was proposed that in a system of acid water in acetonitrile with a ratio $\left[\mathrm{H}^{+}\right] /\left[\mathrm{H}_{2} \mathrm{O}\right]=1: 3$, the proton is mainly found in between two water molecules (not necessarily in a symmetric configuration), forming a Zundel $\mathrm{H}_{5} \mathrm{O}_{2}{ }^{+}$ structure. This finding agrees with the results of earlier linear infrared $^{25}$ and ${ }^{1} \mathrm{H} \mathrm{NMR}^{26}$ studies and a fs-IR study of the proton transfer mode. ${ }^{24}$ Using excitation pulses centered at $2700 \mathrm{~cm}^{-1}$ it was concluded that the excited central $\mathrm{OH}$ stretch vibration of the Zundel-like structure relaxes ultrafast with a time constant $T_{1}<65 \mathrm{fs}^{23}$ The relaxation following excitation at $3400 \mathrm{~cm}^{-1}$ was also observed to be ultrafast with an upper limit for $T_{1}$ of 50 fs. This relaxation behavior was assigned to the $\mathrm{OH}$ vibrations of the two $\mathrm{H}_{2} \mathrm{O}$ molecules flanking the Zundel proton. It was also shown in this study that the fluctuations of the electrical interactions between the hydrated proton and the surrounding acetonitrile molecules strongly modulate the hydration structure geometry and the vibrational potential of the Zundel proton on an ultrafast scale. $^{23,24}$

Ottosson et al. ${ }^{22}$ investigated the picosecond dynamics of hydrated protons in acetonitrile. In this study, it was found that the relaxation of the excited proton partly results in the

Received: March 4, 2019

Revised: June 26, 2019

Published: July 2, 2019 

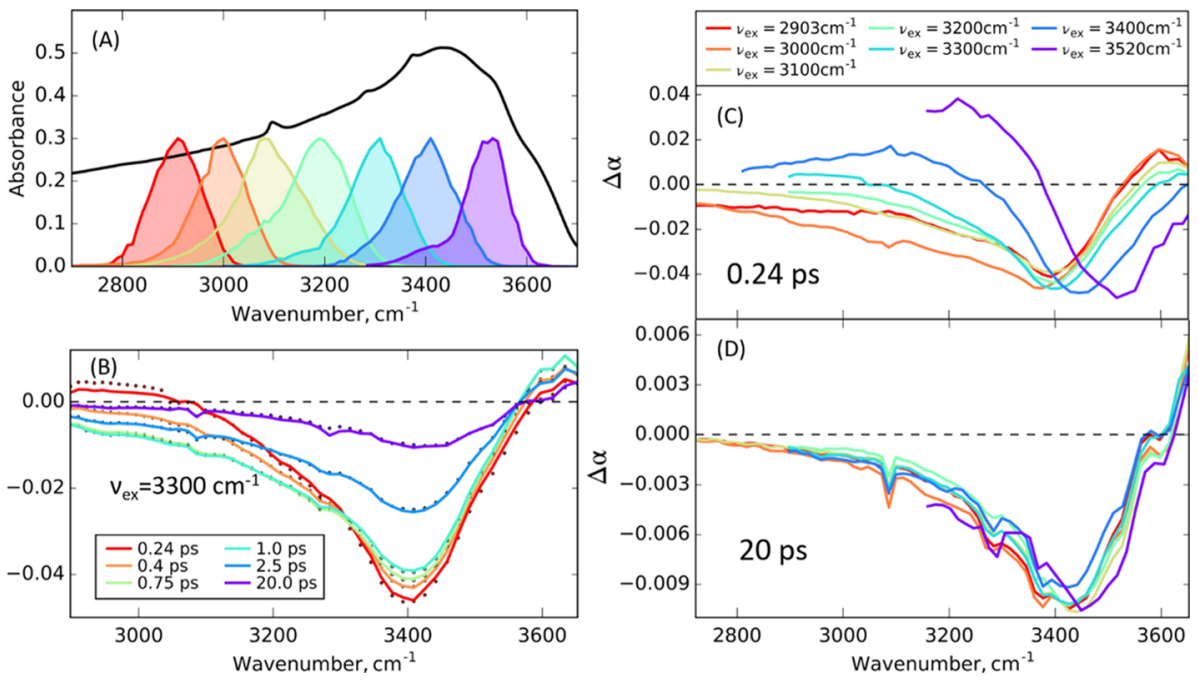

Figure 1. (A) Linear infrared absorption spectrum of the $\mathrm{TfOH} / \mathrm{H}_{2} \mathrm{O}=1: 3$ mixture in acetonitrile- $d_{3}$ (black line, solvent background subtracted) and pump pulses used in the experiments (color lines). (B) Isotropic transient spectra of the hydrated proton in acetonitrile with $\nu_{\mathrm{ex}}=3300 \mathrm{~cm}^{-1}$ (lines represent the result of the fit). (C,D) Isotropic pump-probe spectra at the delay times of 0.24 (C) and 20 ps (D) with the excitation frequency varied.

ultrafast creation $(<100 \mathrm{fs})$ of a locally hot proton hydration cluster, and partly in the vibrational predissociation of a hydrogen bond of the cluster and the release of a water molecule. The re-association of this bond was observed to occur with a time constant of $\sim 6 \mathrm{ps}$.

Here, we present a comparative femtosecond mid-infrared pump-probe spectroscopy study of the vibrational dynamics of the core and outer parts of hydrated proton clusters in acetonitrile. An important difference between the present study and earlier work is that we resolve the frequency-dependent vibrational relaxation time constants of the $\mathrm{OH}$-stretch vibrations of the outer part of the hydration structure. In the work by Dahms et al., ${ }^{23}$ this relaxation was believed to be ultrafast $(<50 \mathrm{fs})$. We find that for excitation frequencies $>3100 \mathrm{~cm}^{-1}$, the relaxation is much slower $(220-370 \mathrm{fs})$ and strongly depends on the frequency, which implies that the $\mathrm{OH}$ stretch vibrational spectrum of the outer part of the proton hydration structure is strongly inhomogeneously broadened.

\section{EXPERIMENT}

We measured transient absorption spectra using an independently tunable femtosecond mid-infrared pump and probe pulses. ${ }^{27}$ The pump and probe pulses are generated using optical parametric amplifiers (OPAs) that are pumped with the $800 \mathrm{~nm} 35 \mathrm{fs}$ pulses produced by a regenerative Ti:sapphire amplifier (Coherent) with $3.5 \mathrm{~mJ}$ energy per pulse. To generate the probe pulses, we pump a homebuilt OPA based on a $\beta$-barium borate $(\mathrm{BBO})$ crystal with $1 \mathrm{~mJ}$ of the $800 \mathrm{~nm}$ pulse energy. The OPA process is seeded with white light. After two amplification stages the OPA delivers the signal and idler pulses with a total energy of $220 \mu \mathrm{J}$. The signal and idler pulses are mixed in a silver gallium disulfide crystal to produce mid-infrared pulses at their difference frequency. The resulting pulses are transmitted through a germanium filter to remove residual signal and idler light, and through calcium fluoride windows to compensate the group velocity dispersion introduced by germanium. The resulting pulses have a central frequency that is tunable in the range $2700-3500 \mathrm{~cm}^{-1}$, a spectral full width at half maximum of $300 \mathrm{~cm}^{-1}$, and a pulse duration of $60 \mathrm{fs}$. The pulses are split into probe and reference pulses using wedged zinc selenide windows. The probe beam was sent through a delay stage to introduce a variable time delay with respect to the pump pulse.

The pump pulses are produced by another OPA based on a $\mathrm{BBO}$ crystal that is pumped with a fraction of $1.3 \mathrm{~mJ}$ of the 800 $\mathrm{nm}$ beam. The resulting idler beam is frequency doubled using another BBO-crystal. Subsequently, the second harmonic of the idler is used as a seed in a second OPA process in a potassium titanyl phosphate crystal that is pumped with a fresh fraction of $1 \mathrm{~mJ}$ of the $800 \mathrm{~nm}$ pulse. This OPA process leads to the amplification of the doubled idler and the production of pulses at the difference frequency. The latter pulses (Figure 1A) are tunable in the range $2900-3500 \mathrm{~cm}^{-1}$ and have a spectral full width at half maximum of $\sim 150 \mathrm{~cm}^{-1}$, a pulse duration of $\sim 120 \mathrm{fs}$, and an energy of $15-20 \mu \mathrm{J}$. The polarization of the pump beam is set at $45^{\circ}$ with respect to the probe polarization using a half-wave plate. The pump pulses are focused into the sample in the spatial overlap with the probe pulses.

After the sample, we select the polarization component of the probe and reference beams that is either parallel or perpendicular to the pump polarization with a polarization filter. Subsequently, the probe and the reference beams are dispersed by a spectrometer and detected by two lines of a $3 \times$ 32 mercury-cadmium-telluride infrared detector array. To obtain transient absorption change spectra $\Delta \alpha_{\|}(\nu, t)$ and $\Delta \alpha_{\perp}(\nu, t)$, every second pump pulse was mechanically chopped. From the measured parallel and perpendicular components, we construct the isotropic signal, which is independent of the transition dipole moment reorientation

$$
\Delta \alpha_{\text {iso }}(\nu, t)=\frac{1}{3}\left(\Delta \alpha_{\|}(\nu, t)+2 \Delta \alpha_{\perp}(\nu, t)\right)
$$

The samples are prepared by dissolving trifluoromethanesulfonic acid (TfOH, 99\%, Sigma-Aldrich) in water and deuterated acetonitrile $\left(\mathrm{CD}_{3} \mathrm{CN}, 99.8 \%\right.$, Sigma-Aldrich $)$ at a ratio $\mathrm{TfOH} / \mathrm{H}_{2} \mathrm{O} / \mathrm{CD}_{3} \mathrm{CN}=1: 3: 75$. Previous IR pumpprobe $^{22}$ and Fourier transform $\mathrm{IR}^{23,25}$ studies showed that the nature of the counter-ion $\left(\mathrm{CF}_{3} \mathrm{SO}_{3}{ }^{-}, \mathrm{ClO}_{4}{ }^{-}\right.$or $\left.\mathrm{I}^{-}\right)$does not have significant influence on the structure and dynamics of the 
hydrated proton in acetonitrile. The solution is put in between two $2 \mathrm{~mm}$ thick calcium fluoride windows separated by a 50 $\mu \mathrm{m}$ Teflon spacer. The nonresonant signal due to the pumpprobe cross-phase modulation in the calcium fluoride windows limits the experimental time resolution to $\sim 0.2 \mathrm{ps}$. In some of the experiments, we obtained a better time resolution by using $500 \mathrm{~nm}$ thick silicon nitride windows instead of calcium fluoride windows.

\section{RESULTS AND DISCUSSION}

Transient Spectra and Delay Time Traces. In Figure $1 \mathrm{~B}$, we show transient absorption spectra following excitation with pulses centered at $3300 \mathrm{~cm}^{-1}$. In Figure 1C,D, we show isotropic transient absorption spectra obtained with different central excitation frequencies at two different delay times $(0.24$ and $20 \mathrm{ps}$ ). At an early delay time of $0.24 \mathrm{ps}$, the transient absorption spectrum strongly depends on the excitation frequency. Excitation with a high-frequency excitation pulse $\left(\nu_{\text {ex }} \geq 3300 \mathrm{~cm}^{-1}\right)$ leads to a small positive absorption change at low frequencies. Excitation at frequencies $\leq 3200 \mathrm{~cm}^{-1}$ leads to a decreased absorption below $3500 \mathrm{~cm}^{-1}$ and an enhanced absorption at probe frequencies $>3500 \mathrm{~cm}^{-1}$. This spectral shape is similar to the transient absorption spectrum observed at long delay times ( $>10$ ps) for all different excitation frequencies. This spectrum consists of a positive absorption change at frequencies $>3600 \mathrm{~cm}^{-1}$ and a broad negative absorption change at lower frequencies.

Coherent Coupling Effects. In a previous study of hydrated protons in acetonitrile an intense increase of the induced absorption within the pump-probe cross-correlation time was observed. ${ }^{23}$ This signal was assigned to Zundel cations excited at $3400 \mathrm{~cm}^{-1}$. Because the observed signal was present only within the cross-correlation of the pump and probe pulses, the authors concluded that the excited state lifetime of these Zundel cations should be below 50 fs. The subsequent slower relaxation was assigned to energy redistribution within the Zundel cation.

In our studies, we observed a similar fast signal component within the pump-probe cross-correlation time. However, we found the contribution of this signal component to be strongly dependent on the difference between the central frequency of the probe pulse and the frequency at which the transient absorption signal was detected. In Figure 2, we show the early delay time dynamics of the induced absorption signal at 3160 $\mathrm{cm}^{-1}$ measured with different probe pulses. After $0.2 \mathrm{ps}$, the transient signal purely consists of the excited state absorption and heating signatures. These signals are independent of the central frequency of the probe pulse. However, in the time interval between -0.1 and $0.2 \mathrm{ps}$, we find that the transient absorption signal becomes increasingly distorted when the detection frequency is shifted further away from the central frequency of the probe pulse. This behavior can be well explained from the additional signal contributions that arise from coherent coupling effects when the pump and probe pulses overlap in time. The origin and impact of these coherent effects are discussed in detail in the Supporting Information.

When the spectrum of the probe pulse is centered at the frequency of maximum of the linear absorption, that is, the frequency of the fundamental $\nu=0 \rightarrow 1$ transition, a strong coherent coupling effect in the region of the excited state absorption (red curve in Figure 2A). This coherent-coupling signal has the form of an additional positive signal and can be easily mistaken for the contribution of an ultrafast decaying
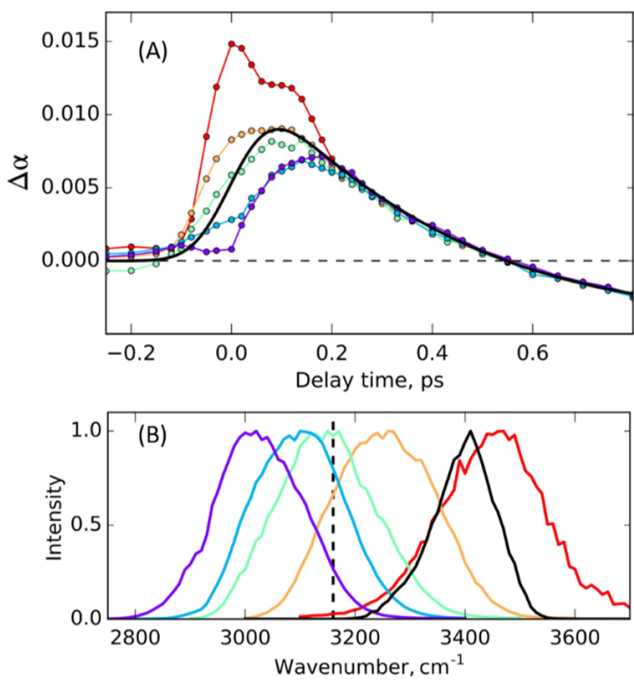

Figure 2. (A) Fast transient absorption dynamics at frequency 3160 $\mathrm{cm}^{-1}$ measured with various probe pulses; the black line represents the fit. (B) Intensity spectra of the probe pulses used (color lines) and the pump pulse (black line).

excited state. We find that this strong additional positive signal vanishes if the spectrum of the probe pulse is centered close to the frequency of interest (green curve in Figure 2A). Therefore, we conclude that excitation at $3400 \mathrm{~cm}^{-1}$ results only in the excitation of relatively slow relaxing water $\mathrm{OH}$ stretch vibrations. In view of the frequency of $3400 \mathrm{~cm}^{-1}$, these $\mathrm{OH}$ vibrations are likely not located in the core of the proton hydration cluster but in the outer part of this structure.

Vibrational Relaxation Dynamics Following Excitation $\leq 3100 \mathrm{~cm}^{-1}$. When we excite the solution with excitation pulses centered at 2900, 3000, and $3100 \mathrm{~cm}^{-1}$, we observe at early delay times a very broad negative signal (bleaching) extending to lower frequencies (Figure 3A). The signal has a zero crossing at $3500 \mathrm{~cm}^{-1}$ and a positive sign (induced absorption) at frequencies $>3500 \mathrm{~cm}^{-1}$. Following
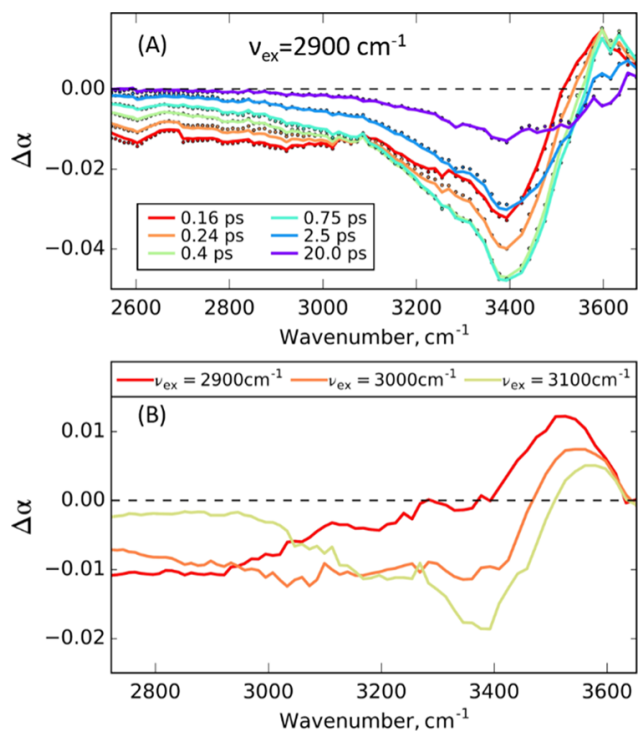

Figure 3. (A) Isotropic transient spectra of the hydrated proton in acetonitrile with $\nu_{\mathrm{ex}}=2900 \mathrm{~cm}^{-1}$ (lines represent the result of the fit). (B) Transient spectral signatures of the local hot state after the low frequency excitation. 
earlier works, ${ }^{22,23}$ we explain the signal at early delay times from a strong local heating effect that results from the ultrafast relaxation of the excited $\mathrm{OH}$-stretch vibrations.

We model the transient spectra obtained with $\nu_{\mathrm{ex}} \leq 3100$ $\mathrm{cm}^{-1}$ with the kinetic model that was used in a previous study of protonated water clusters in acetonitrile. ${ }^{22}$ Within this model the excited vibrational state relaxes to an intermediate state with a relaxation time constant that is too short to be resolved. This intermediate state of the relaxation represents the effect on the absorption of the proton hydration complex of the local dissipation of energy to the water molecules that surround the proton. We will denote this state as the local hot state.

In Figure 3B, we show the transient absorption spectra at early delay times following excitation at 2900, 3000, and 3100 $\mathrm{cm}^{-1}$. The spectral signatures with $\nu_{\mathrm{ex}}=2900$ and $3000 \mathrm{~cm}^{-1}$ consist of a broad negative absorption change below 3400 $\mathrm{cm}^{-1}$ and a positive absorption change around $3500 \mathrm{~cm}^{-1}$. These spectra are similar to the previously obtained spectra with $\nu_{\mathrm{ex}}=2700$ and $2800 \mathrm{~cm}^{-1} \cdot{ }^{22,23}$ The negative part of the spectrum depends on the excitation frequency, which implies that the relaxed energy affects mostly the originally excited vibrations.

The local hot complex relaxes to a second intermediate state that represents the heating of the whole protonated water cluster. The time constant of this relaxation is $0.26 \pm 0.04 \mathrm{ps}$, meaning that the hot proton complex redistributes its energy over the complete protonated water cluster on a time scale of hundreds of femtoseconds. This time constant agrees with the previously reported time constant of 0.32 ps of the energy redistribution following $2800 \mathrm{~cm}^{-1}$ excitation. ${ }^{22}$ In the hot water cluster the hydrogen bonds are weaker, which induces a decrease of the $\mathrm{OH}$-stretch absorption cross-section and a blue shift of the vibrational frequency. On a longer time scale, the hot cluster dissipates its energy to the acetonitrile solvent, resulting in a significant decrease of the amplitude of the heating signal and the appearance of sharp acetonitrile peaks and $\mathrm{OH}$-stretch absorption features that can be assigned to monomeric water molecules.

Vibrational Relaxation Dynamics Following Excitation at Frequencies $\geq 3200 \mathrm{~cm}^{-1}$. For excitation frequencies $\nu_{\mathrm{ex}}>3300 \mathrm{~cm}^{-1}$, we observe a negative absorption change around $3400 \mathrm{~cm}^{-1}$ that we assign to the bleaching of the fundamental $\nu=0 \rightarrow 1$ transition and stimulated $\nu=1 \rightarrow$ 0 emission of the excited $\mathrm{OH}$-stretch vibrations. The positive absorption change below $3200 \mathrm{~cm}^{-1}$ is assigned to the corresponding $\nu=1 \rightarrow 2$ excited state absorption.

The transient absorption spectrum also shows a fast growing spectral component corresponding to the transiently heated hydrated proton cluster. At 1 ps, we observe a broad negative signal with a peak at $\sim 3400 \mathrm{~cm}^{-1}$ and a small induced absorption around $3600 \mathrm{~cm}^{-1}$ (see Figure 1B). This spectrum has a very similar shape as the spectrum that is observed after 1 ps for excitation frequencies $\nu_{\text {ex }} \leq 3100 \mathrm{~cm}^{-1}$, and that is observed at much later delay times, for example at 20 ps. Hence, we assign this spectrum to the response of the complete hot protonated water cluster.

Because of its large transient spectral amplitude, the spectral response of the hot protonated water cluster becomes significant already at early delay times and dominates the initial positive absorption signal in the region of the $\nu=1 \rightarrow 2$ excited state absorption. The transient signal at $\nu_{\mathrm{pr}}=3000$ $\mathrm{cm}^{-1}$ becomes negative at $0.7 \mathrm{ps}$ for $\nu_{\mathrm{ex}}=3400 \mathrm{~cm}^{-1}$, and already at $0.3 \mathrm{ps}$ for $\nu_{\mathrm{ex}}=3300 \mathrm{~cm}^{-1}$ (Figure 4A). The transient absorption signal at $\nu_{\mathrm{pr}}=3400 \mathrm{~cm}^{-1}$ (Figure 4B)

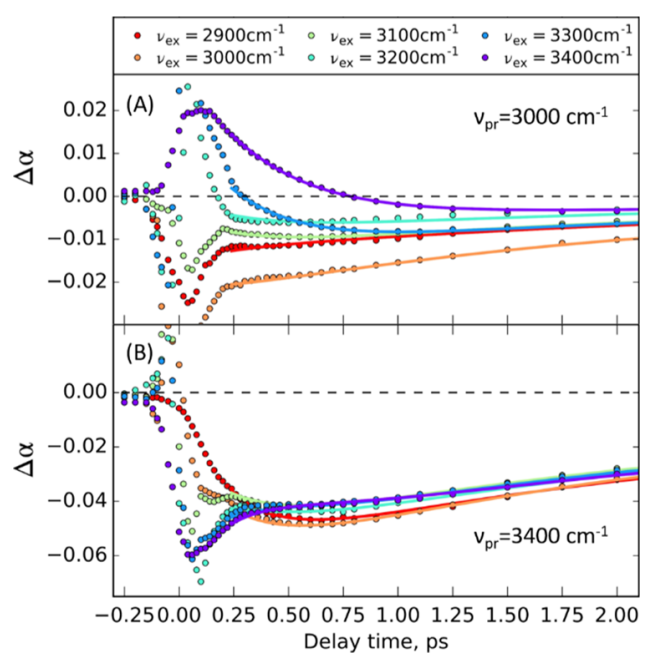

Figure 4. Isotropic pump-probe signal of the hydrated proton in acetonitrile at frequencies 3000 (A) and $3400 \mathrm{~cm}^{-1}$ (B) as a function of delay time; excitation frequency varies from 2900 to $3400 \mathrm{~cm}^{-1}$. Lines represent the result of the fit.

shows a much slower decay because at this frequency, the initial signal has a negative sign as it is due to the $\nu=0 \rightarrow 1$ bleaching and stimulated $\nu=1 \rightarrow 0$ emission. At this detection frequency the vibrational relaxation process replaces this initial negative signal by the negative absorption change associated with the hot cluster.

We fit the experimental data obtained with excitation frequencies $\geq 3300 \mathrm{~cm}^{-1}$ with a kinetic model that contains four states. The first state is the $\nu=1$ state of the $\mathrm{OH}$-stretch vibration that is excited by the excitation pulse. The relaxation of this state results in heating of the protonated water cluster. We observe that the rise of this heating is somewhat delayed with respect to the relaxation of the excited $\nu=1$ state. It has been observed before in studies of the vibrational relaxation of bulk water that the heating signal grows somewhat slower than the excited vibration relaxes. ${ }^{28,29}$ This delay is not observed when we excite low-frequency $\mathrm{OH}$-stretch vibrations with $\nu_{\text {ex }}$ $\leq 3100 \mathrm{~cm}^{-1}$ (the local hot state is observed immediately), which indicates that the low- and high-frequency $\mathrm{OH}$ vibration have different relaxation mechanisms. The relaxation of the high-frequency $\mathrm{OH}$ vibration likely proceeds through an intermediate state with an associated response that is quite different from a local heating effect. To account for the delay of the heating effect following the relaxation of the highfrequency $\mathrm{OH}$-stretch vibrations, we include in the model an intermediate state in between the excited $\nu=1$ state and the hot water cluster state. We did not include the relatively small and long living spectral component of the vibrational predissociation that has been observed before. ${ }^{22}$ The hot cluster exchanges heat energy with its surrounding to reach the fourth state in the model which represents the eventual globally heated state of the system.

The relaxation dynamics of the $\mathrm{OH}$-stretch excited state can be well estimated from the low frequency region, where $\nu=1$ $\rightarrow 2$ excited state absorption is the dominant spectral component. Comparing the transient absorption dynamics in this frequency region (Figure 4A), we observe a strong difference between the results of excitation at $3400 \mathrm{~cm}^{-1}$ and at 
$3300 \mathrm{~cm}^{-1}$. The initial signal value at $\nu_{\mathrm{pr}}=3000 \mathrm{~cm}^{-1}$ is much smaller when the oscillators are excited at the lower frequency. This observation cannot be explained from a much faster relaxation of the $\mathrm{OH}$-stretch excited state, because the dynamics are similar to what is observed in the case of excitation at $3400 \mathrm{~cm}^{-1}$. The observation of a small induced absorption signal at early delay times indicates that the hot cluster state is in part directly populated, that is, in a separate relaxation channel that is faster than our time resolution. We thus conclude that the excitation pulse centered at $3300 \mathrm{~cm}^{-1}$ excites two types of $\mathrm{OH}$ oscillators. We find a good description of the transient spectra observed for $\nu_{\mathrm{ex}}=3300 \mathrm{~cm}^{-1}$ when 40 $\pm 10 \%$ of the excited $\mathrm{OH}$ vibrations relax very rapidly $\left(T_{1}<50\right.$ fs) and directly populate the hot cluster state, and $60 \pm 10 \%$ relaxes more slowly with a time constant of $270 \pm 30$ fs. For $\nu_{\text {ex }}$ $=3200 \mathrm{~cm}^{-1}$, we find that $70 \pm 10 \%$ relaxes directly to the hot cluster state and $30 \pm 10 \%$ relaxes with a time constant of 220 \pm 40 fs.

In Figure 5A, we show the spectral signatures of the excited $\nu=1$ state extracted from the fit. The spectrum of the excited
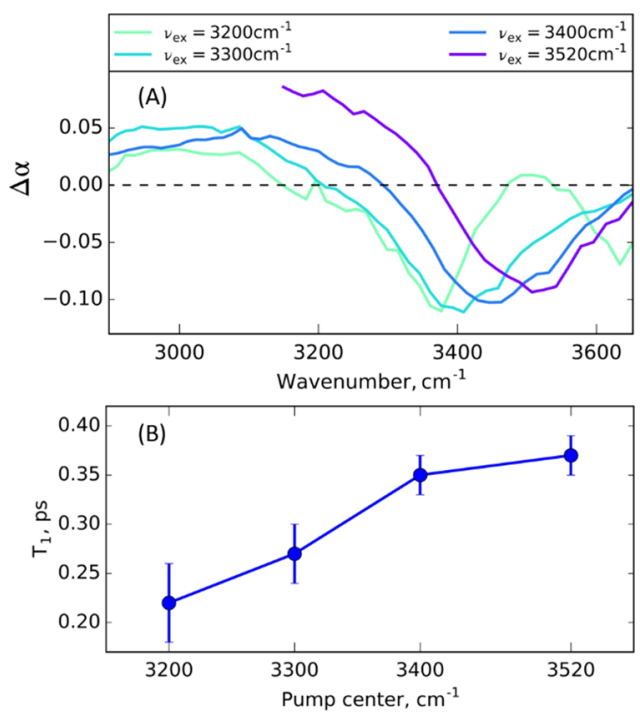

Figure 5. (A) Transient spectra associated with the excitation of the $\nu$ $=1$ state of the $\mathrm{OH}$-stretch vibration for four different excitation frequencies. (B) Time constant $T_{1}$ of the relaxation of the $\nu=1$ state as a function of the central excitation frequency.

state shows a significant dependence on the excitation frequency: the position of the ground state bleach shifts from 3500 to $3380 \mathrm{~cm}^{-1}$, and the excited state lifetime $T_{1}$ decreases from $370 \pm 20 \mathrm{fs}$ at $\nu_{\mathrm{ex}}=3520 \mathrm{~cm}^{-1}$ to $220 \pm 40 \mathrm{fs}$ at $\nu_{\mathrm{ex}}=3200 \mathrm{~cm}^{-1}$ (Figure $5 \mathrm{~B}$ ).

The observed frequency dependence of the relaxation time constant indicates that the spectrum of the $\mathrm{OH}$-stretch vibrations is inhomogeneously broadened. The variation of the excited state lifetime $T_{1}$ correlates well with the variation in local hydrogen-bond strength: $\mathrm{OH}$-groups engaged in stronger hydrogen bonds have lower absorption frequencies and show faster relaxation. Interestingly, the dependence of the vibrational lifetime on the excitation frequency is very similar to that observed for bulk liquid water, ${ }^{30}$ for which $T_{1}$ was found to decrease from $0.4 \mathrm{ps}$ for $\nu_{\mathrm{ex}}=3500 \mathrm{~cm}^{-1}$ to $0.25 \mathrm{ps}$ for $\nu_{\mathrm{ex}}=$ $3200 \mathrm{~cm}^{-1}$.

\section{DISCUSSION}

We observe strongly the different early delay time transient spectra when we shift the excitation frequency from 2900 to $3520 \mathrm{~cm}^{-1}$. This finding shows that the $\mathrm{OH}$-stretch vibrational spectrum represents $\mathrm{OH}$-groups of highly different characters. At frequencies $\leq 3100 \mathrm{~cm}^{-1}$, we excite $\mathrm{OH}$ vibrations located in the core of the proton hydration cluster. For the (distorted) Zundel structure, this core is formed by the $\mathrm{OH}$-stretch vibration involving the central $\mathrm{H}$ atom in the $\mathrm{H}_{5} \mathrm{O}_{2}{ }^{+}$structure, for the Eigen structure the core is formed by the $\mathrm{OH}$ vibrations involving the three $\mathrm{H}$ atoms of the central $\mathrm{H}_{3} \mathrm{O}^{+}$of the $\mathrm{H}_{9} \mathrm{O}_{4}^{+}$ structure. The main property of these $\mathrm{OH}$ vibrations that the $\mathrm{H}$ atom carries is a significant part of the positive charge, and as a result donates a strong hydrogen bond. As a result, the frequency of these $\mathrm{OH}$ vibrations is $<3200 \mathrm{~cm}^{-1}$ and the vibrational relaxation is ultrafast $(<50 \mathrm{fs})$.

For excitation frequencies $>3200 \mathrm{~cm}^{-1}$, the observed transient spectra resemble that of the $\mathrm{OH}$-stretch vibration in neat water and hydrogen-bonded water in other media. The vibrational relaxation of this excited state is about 30 times faster than that of the isolated water molecules in acetonitrile $\left(T_{1}=8 \mathrm{ps}\right),{ }^{31,32}$ and has a quite similar relaxation rate as the $\mathrm{OH}$-stretch vibrations in bulk water. ${ }^{30}$ From this we conclude that the observed high frequency $\mathrm{OH}$-stretch vibrations belong to water molecules of a cluster that contains at least one proton, but that is not in the center of the proton hydration structure, that is, the $\mathrm{H}$ atoms involved in these $\mathrm{OH}$ vibrations do not carry a significant amount of the positive proton charge. At a concentration ratio of $\left[\mathrm{H}^{+}\right] /\left[\mathrm{H}_{2} \mathrm{O}\right]=1: 3$, the hydrated proton clusters in acetonitrile contain $2-6$ water molecules. ${ }^{26}$ The $\mathrm{OH}$ vibrations giving rise to this response can thus belong to the two $\mathrm{H}_{2} \mathrm{O}$ molecules flanking the Zundel proton, or the $\mathrm{OH}$ vibrations of the three outer $\mathrm{H}_{2} \mathrm{O}$ molecules of the Eigen $\mathrm{H}_{9} \mathrm{O}_{4}{ }^{+}$structure, or water molecules even further away from the core of the proton hydration structure. All these $\mathrm{OH}$ vibrations have in common that the partial positive charge on the $\mathrm{H}$ atom is small and that they absorb at frequencies $\geq 3200$ $\mathrm{cm}^{-1}$.

Excitation at intermediate frequencies of $3100-3200 \mathrm{~cm}^{-1}$ yields spectral dynamics of the intermediate character. We observe $\mathrm{OH}$-stretch vibrations showing a fast but resolvable vibrational relaxation, and the direct creation of a local hot state. It thus follows that we observe quite a distinct vibrational relaxation behavior of the core and the outer part of the proton hydration cluster, even in the spectral region where the spectra of the corresponding $\mathrm{OH}$ vibrations overlap.

Interestingly, the transient spectrum observed at long delay times and that we assign to the response of the entire hot water cluster are the same irrespective of the original excitation frequency. This finding shows that all $\mathrm{OH}$-stretch vibrations absorbing in the region from 3520 to $2900 \mathrm{~cm}^{-1}$ belong to protonated water clusters that have similar absorption spectra and are thus likely of similar composition.

Combining the results of all different excitation pulses, we summarize the relaxation processes of the different $\mathrm{OH}$-stretch vibrations in the protonated water cluster with the kinetic scheme shown in Figure 6. In this scheme, the $\nu=1$ state relaxes to a local hot state with a time constant $T_{1}$ that decreases with the decreasing excitation frequency. The high frequency part $\left(\geq 3200 \mathrm{~cm}^{-1}\right)$ of the spectrum corresponds to the $\mathrm{OH}$-stretch vibrations of the water molecules in the outer part of the proton hydration cluster, which display the 


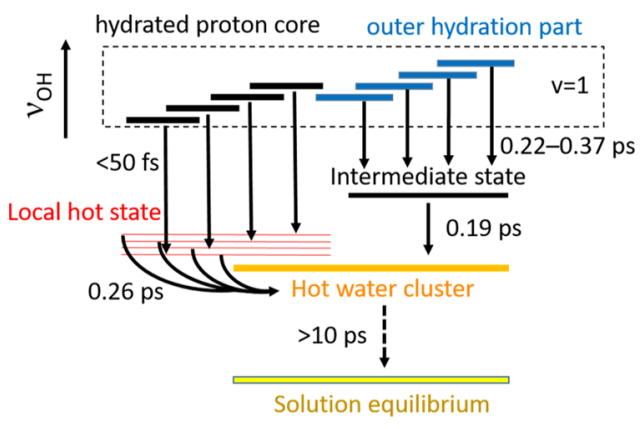

Figure 6. Kinetic scheme describing the spectral dynamics after excitation of the hydrated proton $\mathrm{OH}$ vibrations.

vibrational relaxation dynamics similar to that of bulk water. The lower frequency absorption of the protonated cluster is due to the vibrations of the core of the cluster, the excited state of which relaxes much faster. The absorption spectra of these two types of vibrations overlap in the frequency region of $3100-3300 \mathrm{~cm}^{-1}$, and excitation pulses in this frequency region will thus excite both the core and the outer part of the proton hydration cluster. As a result, we observe mixed vibrational relaxation dynamics. For all $\mathrm{OH}$ vibrations, the relaxation results in a local hot state with a spectrum that depends on the excitation frequency. At lower frequencies, this spectrum will primarily reflect the effect of local heat dissipation on the core of the proton hydration structure. At higher frequencies, the spectrum of the local hot state is blueshifted and is very similar to that of the complete hot water cluster, that is, the state that results after the heat has been equilibrated over the entire proton hydration cluster.

We note that the transient hot states observed in our experiments are very local in nature and differ from a true thermal state of the whole sample. As a result, these transient hot spectra differ from the linear thermal difference spectrum of the hydrated proton in acetonitrile. This latter spectrum shows a much broader negative absorption change than that observed in the femtosecond experiments at delay time $>10$ ps. $^{22}$ An increase in the equilibrium temperature is observed to lead to a similar decrease of the cross-section for all $\mathrm{OH}$ stretch vibrations (see Supporting Information). In contrast, the pump-probe spectrum at 1 ps shows predominant absorption changes in the frequency region of the outer part of the cluster even when the core of the cluster was initially excited. Apparently, the studied system does not reach a complete thermal equilibrium within the picosecond time frame of the pump-probe experiment. Similar observations were done for pump-probe experiments on the hydrated proton in dimethyl sulfoxide ${ }^{27}$ and in bulk water. ${ }^{33,34}$ This discrepancy between the final thermal difference spectrum of the pump-probe experiment and the fully equilibrated response to heating of the sample can be explained from the fact that complete equilibration includes a change of the composition of the clusters. This change in composition involves diffusion and exchange of molecules between different clusters. These processes occur on much longer time scales than the picosecond times scale of the time-resolved experiments.

\section{CONCLUSIONS}

We studied the vibrational relaxation and energy dissipation of small protonated water clusters in acetonitrile using femtosecond mid-IR pump-probe spectroscopy. We find that the relaxation dynamics strongly depend on the excitation frequency. Excitation of the low frequency part of the $\mathrm{OH}$ stretch band $\left(\leq 3100 \mathrm{~cm}^{-1}\right)$ is followed by the ultrafast vibrational relaxation $\left(T_{1}<50 \mathrm{fs}\right)$, resulting in a local energy dissipation that affects mostly the originally excited vibrations. The transient spectrum of this local hot state shows a broad negative absorption change around the excitation frequency. After the fast vibrational relaxation of these $\mathrm{OH}$ groups, the energy equilibrates over the complete protonated water cluster with a time constant of $0.26 \pm 0.04$ ps.

Excitation of the high-frequency part of the $\mathrm{OH}$-stretch band $\left(\leq 3200 \mathrm{~cm}^{-1}\right)$ is followed by vibrational relaxation of the $\mathrm{OH}$ stretch vibration with a time constant ranging from $T_{1}=0.22$ \pm 0.04 ps for an excitation pulse centered at $3200 \mathrm{~cm}^{-1}$ to $T_{1}=$ $0.37 \pm 0.02$ ps for an excitation pulse centered at $3520 \mathrm{~cm}^{-1}$. We found that the signal previously assigned to the relaxation of the Zundel cation excited at $3400 \mathrm{~cm}^{-1}$ strongly depends on the probe pulse and originates from the coherent coupling of pump and probe pulses. The vibrational relaxation results in a somewhat delayed heating of the complete protonated water cluster, which indicates that the high-frequency $\mathrm{OH}$-stretch vibrations have a different relaxation mechanism, that is, it proceeds through a different intermediate state in comparison with the low-frequency $\mathrm{OH}$-stretch vibrations.

The spectrum of the hot protonated water cluster that results after relaxation of the high-frequency $\mathrm{OH}$ vibrations is the same as those observed after excitation of the lowfrequency $\mathrm{OH}$-stretch vibrations. This result shows that the high- and low-frequency $\mathrm{OH}$ vibrations belong to the same type of protonated water clusters. We thus assign the lowfrequency part of the $\mathrm{OH}$ spectrum to the core of the proton hydration cluster and the high-frequency part of this spectrum to the outer part of this cluster. At intermediate excitation frequencies between 3100 and $3300 \mathrm{~cm}^{-1}$, we do not observe average or intermediate behavior but two distinct relaxation components. Part of the $\mathrm{OH}$ vibrations show the ultrafast relaxation behavior of the core while the other part shows the relatively slower relaxation dynamics of the outer part. This latter fraction increases with the increasing excitation frequency. We conclude that the core shows quite distinct vibrational relaxation behavior from the outer part, even in the spectral region where the spectra of the corresponding $\mathrm{OH}$ vibrations overlap.

\section{ASSOCIATED CONTENT}

\section{S Supporting Information}

The Supporting Information is available free of charge on the ACS Publications website at DOI: 10.1021/acs.jpcb.9b02067.

Discussion of the impact of the coherent effects on the measured pump-probe signal and comparison of the linear thermal difference spectrum and the pump-probe spectra at different delay times (PDF)

\section{AUTHOR INFORMATION}

\section{Corresponding Author}

*E-mail: sofronov@amolf.nl.

ORCID ${ }^{\circ}$

Oleksandr O. Sofronov: 0000-0001-7744-6404

Huib J. Bakker: 0000-0003-1564-5314

Notes

The authors declare no competing financial interest. 


\section{ACKNOWLEDGMENTS}

This work is part of the research program of the Netherlands Organization for Scientific Research (NWO) and was performed at the research institute AMOLF. This project has received funding from the European Research Council (ERC) under the European Union's Horizon 2020 research and innovation program (grant agreement no. 694386). The authors thank Dr. Jan Versluis and Hinco Shoenmaker for the experimental support.

\section{REFERENCES}

(1) Kreuer, K.-D. Proton Conductivity: Materials and Applications. Chem. Mater. 1996, 8, 610-641.

(2) Garczarek, F.; Gerwert, K. Functional Waters in Intraprotein Proton Transfer Monitored by FTIR Difference Spectroscopy. Nature 2006, 439, 109-112.

(3) Voth, G. A. Computer Simulation of Proton Solvation and Transport in Aqueous and Biomolecular Systems. Acc. Chem. Res. 2006, 39, 143-150.

(4) Agmon, N.; Bakker, H. J.; Campen, R. K.; Henchman, R. H.; Pohl, P.; Roke, S.; Thämer, M.; Hassanali, A. Protons and Hydroxide Ions in Aqueous Systems. Chem. Rev. 2016, 116, 7642-7672.

(5) Headrick, J. M.; Diken, E. G.; Walters, R. S.; Hammer, N. I.; Christie, R. A.; Cui, J.; Myshakin, E. M.; Duncan, M. A.; Johnson, M. A.; Jordan, K. D. Spectral Signatures of Hydrated Proton Vibrations in Water Clusters. Science 2005, 308, 1765-1769.

(6) Wolke, C. T.; Fournier, J. A.; Dzugan, L. C.; Fagiani, M. R.; Odbadrakh, T. T.; Knorke, H.; Jordan, K. D.; McCoy, A. B.; Asmis, K. R.; Johnson, M. A. Spectroscopic Snapshots of the Proton-Transfer Mechanism in Water. Science 2016, 354, 1131-1135.

(7) Marx, D.; Tuckerman, M. E.; Hutter, J.; Parrinello, M. The Nature of the Hydrated Excess Proton in Water. Nature 1999, 397, 601-604.

(8) Kulig, W.; Agmon, N. A "clusters-in-Liquid" Method for Calculating Infrared Spectra Identifies the Proton-Transfer Mode in Acidic Aqueous Solutions. Nat. Chem. 2013, 5, 29-35.

(9) Markovitch, O.; Chen, H.; Izvekov, S.; Paesani, F.; Voth, G. A.; Agmon, N. Special Pair Dance and Partner Selection: Elementary Steps in Proton Transport in Liquid Water. J. Phys. Chem. B 2008, 112, 9456-9466.

(10) Śmiechowski, M.; Stangret, J. Proton Hydration in Aqueous Solution: Fourier Transform Infrared Studies of HDO Spectra. J. Chem. Phys. 2006, 125, 204508.

(11) Stoyanov, E. S.; Stoyanova, I. V.; Reed, C. A. The unique nature of $\mathrm{H}+$ in water. Chem. Sci. 2011, 2, 462-472.

(12) Fournier, J. A.; Wolke, C. T.; Johnson, C. J.; Johnson, M. A.; Heine, N.; Gewinner, S.; Schöllkopf, W.; Esser, T. K.; Fagiani, M. R.; Knorke, $\mathrm{H}_{\text {.; }}$ et al. Site-specific vibrational spectral signatures of water molecules in the magic $\mathrm{H}_{3} \mathrm{O}^{+}\left(\mathrm{H}_{2} \mathrm{O}\right)_{20}$ and $\mathrm{Cs}^{+}\left(\mathrm{H}_{2} \mathrm{O}\right)_{20}$ clusters. Proc. Natl. Acad. Sci. U.S.A. 2014, 111, 18132-18137.

(13) Fagiani, M. R.; Knorke, H.; Esser, T. K.; Heine, N.; Wolke, C. T.; Gewinner, S.; Schöllkopf, W.; Gaigeot, M.-P.; Spezia, R.; Johnson, M. A.; et al. Gas Phase Vibrational Spectroscopy of the Protonated Water Pentamer: The Role of Isomers and Nuclear Quantum Effects. Phys. Chem. Chem. Phys. 2016, 18, 26743-26754.

(14) Markovitch, O.; Agmon, N. Structure and Energetics of the Hydronium Hydration Shells. J. Phys. Chem. A 2007, 111, 22532256.

(15) Marx, D.; Chandra, A.; Tuckerman, M. E. Aqueous Basic Solutions: Hydroxide Solvation, Structural Diffusion, and Comparison to the Hydrated Proton. Chem. Rev. 2010, 110, 2174-2216.

(16) Biswas, R.; Tse, Y.-L. S.; Tokmakoff, A.; Voth, G. A. Role of Presolvation and Anharmonicity in Aqueous Phase Hydrated Proton Solvation and Transport. J. Phys. Chem. B 2016, 120, 1793-1804.

(17) Nibbering, E. T. J.; Elsaesser, T. Ultrafast Vibrational Dynamics of Hydrogen Bonds in the Condensed Phase. Chem. Rev. 2004, 104, $1887-1914$.
(18) Kim, J.; Schmitt, U. W.; Gruetzmacher, J. A.; Voth, G. A.; Scherer, N. E. The Vibrational Spectrum of the Hydrated Proton: Comparison of Experiment, Simulation, and Normal Mode Analysis. J. Chem. Phys. 2002, 116, 737-746.

(19) Biswas, R.; Carpenter, W.; Fournier, J. A.; Voth, G. A.; Tokmakoff, A. IR Spectral Assignments for the Hydrated Excess Proton in Liquid Water. J. Chem. Phys. 2017, 146, 154507.

(20) Xu, J.; Zhang, Y.; Voth, G. A. Infrared Spectrum of the Hydrated Proton in Water. J. Phys. Chem. Lett. 2011, 2, 81-86.

(21) Napoli, J. A.; Marsalek, O.; Markland, T. E. Decoding the Spectroscopic Features and Time Scales of Aqueous Proton Defects. J. Chem. Phys. 2018, 148, 222833.

(22) Ottosson, N.; Liu, L.; Bakker, H. J. Vibrational Relaxation of the Aqueous Proton in Acetonitrile: Ultrafast Cluster Cooling and Vibrational Predissociation. J. Phys. Chem. B 2016, 120, 7154-7163.

(23) Dahms, F.; Costard, R.; Pines, E.; Fingerhut, B. P.; Nibbering, E. T. J.; Elsaesser, T. The Hydrated Excess Proton in the Zundel Cation $\mathrm{H}_{5} \mathrm{O}_{2}{ }^{+}$: The Role of Ultrafast Solvent Fluctuations. Angew. Chem., Int. Ed. 2016, 55, 10600-10605.

(24) Dahms, F.; Fingerhut, B. P.; Nibbering, E. T. J.; Pines, E.; Elsaesser, T. Large-Amplitude Transfer Motion of Hydrated Excess Protons Mapped by Ultrafast 2D IR Spectroscopy. Science 2017, 357, 491-495.

(25) Kalish, N. B.-M.; Shandalov, E.; Kharlanov, V.; Pines, D.; Pines, E. Apparent Stoichiometry of Water in Proton Hydration and Proton Dehydration Reactions in $\mathrm{CH}_{3} \mathrm{CN} / \mathrm{H}_{2} \mathrm{O}$ Solutions. J. Phys. Chem. A 2011, 115, 4063-4075.

(26) Sigalov, M. V.; Kalish, N.; Carmeli, B.; Pines, D.; Pines, E. Probing Small Protonated Water Clusters in Acetonitrile Solutions By ${ }^{1}$ H NMR. Z. Phys. Chem. 2013, 227, 983-1007.

(27) Sofronov, O. O.; Bakker, H. J. Energy Relaxation and Structural Dynamics of Protons in Water/DMSO Mixtures. J. Phys. Chem. B 2018, 122, 10005-10013.

(28) Ramasesha, K.; De Marco, L.; Mandal, A.; Tokmakoff, A. Water Vibrations Have Strongly Mixed Intra- and Intermolecular Character. Nat. Chem. 2013, 5, 935-940.

(29) Hunger, J.; Liu, L.; Tielrooij, K.-J.; Bonn, M.; Bakker, H. Vibrational and Orientational Dynamics of Water in Aqueous Hydroxide Solutions. J. Chem. Phys. 2011, 135, 124517.

(30) van der Post, S. T.; Hsieh, C. S.; Okuno, M.; Nagata, Y.; Bakker, H. J.; Bonn, M.; Hunger, J. Strong Frequency Dependence of Vibrational Relaxation in Bulk and Surface Water Reveals SubPicosecond Structural Heterogeneity. Nat. Commun. 2015, 6, 8384.

(31) Cringus, D.; Jansen, T. L. C.; Pshenichnikov, M. S.; Wiersma, D. A. Ultrafast Anisotropy Dynamics of Water Molecules Dissolved in Acetonitrile. J. Chem. Phys. 2007, 127, 084507.

(32) Cringus, D.; Yeremenko, S.; Pshenichnikov, M. S.; Wiersma, D. A. Hydrogen Bonding and Vibrational Energy Relaxation in WaterAcetonitrile Mixtures. J. Phys. Chem. B 2004, 108, 10376-10387.

(33) Carpenter, W. B.; Fournier, J. A.; Lewis, N. H. C.; Tokmakoff, A. Picosecond Proton Transfer Kinetics in Water Revealed with Ultrafast IR Spectroscopy. J. Phys. Chem. B 2018, 122, 2792-2802.

(34) Thämer, M.; De Marco, L.; Ramasesha, K.; Mandal, A.; Tokmakoff, A. Ultrafast 2D IR Spectroscopy of the Excess Proton in Liquid Water. Science 2015, 350, 78-82. 